\title{
Mechanisms of carrier transport induced by a microswimmer bath
}

\author{
Andreas Kaiser, ${ }^{1,-\circledast}$ Andrey Sokolov, ${ }^{2}$ Igor S. Aranson, ${ }^{2}$ and Hartmut Löwen ${ }^{1}$ \\ ${ }^{1}$ Institut für Theoretische Physik II: Weiche Materie, \\ Heinrich-Heine-Universität Düsseldorf, Universitätsstraße 1, D-40225 Düsseldorf, Germany \\ ${ }^{2}$ Materials Science Division, Argonne National Laboratory, \\ 9700 South Cass Avenue, Argonne, Illinois 60439, USA
}

(Dated: April 27, 2022)

\begin{abstract}
Recently, it was found that a wedgelike microparticle (referred to as "carrier") which is only allowed to translate but not to rotate exhibits a directed translational motion along the wedge cusp if it is exposed to a bath of microswimmers. Here we model this effect in detail by resolving the microswimmers explicitly using interaction models with different degrees of mutual alignment. Using computer simulations we study the impact of these interactions on the transport efficiency of V-shaped carrier. We show that the transport mechanisms itself strongly depends on the degree of alignment embodied in the modelling of the individual swimmer dynamics. For weak alignment, optimal carrier transport occurs in the turbulent microswimmer state and is induced by swirl depletion inside the carrier. For strong aligning interactions, optimal transport occurs already in the dilute regime and is mediated by a polar cloud of swimmers in the carrier wake pushing the wedge-particle forward. We also demonstrate that the optimal shape of the carrier leading to maximal transport speed depends on the kind of interaction model used.
\end{abstract}

\section{INTRODUCTION}

The collective properties of active fluids have been studied intensively in the last years [1-4]. Examples of such active systems can be found in quite different areas of nature ranging from bacteria [5], alga 9 -11], spermatozoa 12 14], animals 15] - like birds [16], fish [17] or insects [18, 19] - and even human beings [20 22]. All of these systems can be categorized as living active systems. Additionally, there are a broad class of artificial realizations, based on various propulsion mechanisms like pure body rotation [23], propulsion by attached flagella 2426] or various chemically induced mechanisms like selfdiffusiophoresis [27 32] or self-thermophoresis [33 35].

All of these swimmers are known to form spatiotemporal active states like swarming [36 39] and turbulence (swirling) [40 48]. Most of these patterns can be obtained by a simple modeling based on excluded volume effects of effective anisotropic objects [42, 49]. However, the actual particle collisions and the degree of alignment are supposed to play an important role [50, 51]. A mutual alignment of colliding swimmers provides a mechanism of swarming 52, as observed for a lot of artificial active systems [51, 53 55]. On the other hand experiments have shown a swim-off effect of two bacteria after the collisions [5, [56, 57].

In the last years, in experiment as well as in simulations, active fluids have been considered in the presence of passive objects or obstacles. Fixed boundaries have been shown to guide active particles [58, 59] and accumulate them 60 64. This effect has been used to rectify the motion of swimmers [65 69], and building sorting 70 73] as well as trapping devices for microswimmers [59, 74, 75].

\footnotetext{
* kaiser@thphy.uni-duesseldorf.de
}

The motion of passive particles submersed in complex systems 76], like active fluids, has been studied as well. Starting with simple small spherical [77-79] and curved [80] tracer particles to large deformable chains [81], showing a regime of ballistic motion as known for active particles themselves [29, 82, 83].

Recently, it has been shown that energy can be extracted from active fluids and biomolecular motors [84]. Asymmetric cogwheels submersed in an active bath spontaneously rotate 85 88]. Moreover, it has been demonstrated recently that wedgelike microparticles can be transported in the highly dilute [89] and in the turbulent state [90] of the active bath.

Here, we focus on the modelling of the dynamics of a wedgelike microparticle (referred to in the following as "carrier") when it is exposed to a bath of microswimmers. These V-shape particles can be fabricated by photolithography [35, 86, 91] and can be submersed into a bath of biological or artificial microswimmers. By using an external field, the carrier is only allowed to translate but not to rotate. In this paper, we resolve the microswimmers explicitly using different interaction models with different degrees of alignment after a binary collision. We study the impact of these interactions on the transport efficiency of the carrier in detail and show that the transport mechanisms itself strongly depends on the degree of alignment embodied in the modelling of the individual swimmer dynamics. In the modelling so far [90], the maximal carrier speed occurred in the turbulent state and caused by swirl depletion. Here we show that for strongly aligning interactions the picture is different: an even higher transport speed can be achieved in a dilute active fluid which is induced by a smectic ordered cluster in the wake of the carrier. In contrast to earlier work [89], here a full swarm has developed to push the carrier in an efficient way. We also compute the shape of the carrier maximizing the transport speed and show that it depends 
on the kind of interaction model used.

This paper is organized as follows: First we specify our modelling in Sec. III before we study and compare the collective behavior in Sec. [II] In Sec. IV] we study the transport efficiency of a wedgelike carrier and work out the underlying mechanisms, which are directly linked to swimmer-swimmer interactions. Furthermore, we compute the optimal shape of the carrier which leads to the maximum transport speed. Finally, we conclude in Sec. V

\section{MODEL}

We model the active bath in two spatial dimensions by considering $N$ rodlike self-propelled particles with center-of-mass positions $\mathbf{r}_{\alpha}$ and orientations $\hat{\mathbf{u}}_{\alpha}(\alpha=$ $1, \ldots, N)$, using a possible effective body shape asymmetry analogous to Ref. [90] in the absence of noise and hydrodynamic interactions. Therefore, each rod of length $\ell$ and width $\lambda$ is discretized into $n=6$ spherical segments equidistantly positioned along the main $\operatorname{rod}$ axis $\hat{\mathbf{u}}=(\cos \varphi, \sin \varphi)$. The aspect ratio of the swimmers is fixed to $\ell / \lambda=5$ according to a previous work regarding the explicit realization Bacillus subtilis. Between the segments of different rods a repulsive Yukawa potential is imposed [92]. The resulting total pair potential of a rod pair $\alpha, \beta$ is given by $U_{\alpha \beta}=\sum_{i=1}^{n} \sum_{j=1}^{n} U_{i} U_{j} \exp \left[-r_{i j}^{\alpha \beta} / \lambda\right] / r_{i j}^{\alpha \beta}$ where $\lambda$ is the screening length defining the particle diameter, and $r_{i j}^{\alpha \beta}=\left|\mathbf{r}_{i}^{\alpha}-\mathbf{r}_{j}^{\beta}\right|$ the distance between segment $i$ of $\operatorname{rod} \alpha$ and $j$ of $\operatorname{rod} \beta(\alpha \neq \beta)$. The effective body shape of the rods can be tuned by the interaction prefactor of the first segment of each rod with respect to the others, see Fig. 1. This quantity will be given by the ratio $U^{*}=U_{1}^{2} / U_{j}^{2}$ $(j=2 \ldots n)$, where $U^{*}=1$ refers to a symmetric rod. Any overlap of particles is avoided by imposing a large interaction strength $U_{j}^{2}=2.5 F_{0} \ell$. The shape asymmetry allows us to controll the degree of alignment during a binary rod-rod collision [93]. Here we study two situations in detail: first of all, the symmetric case where $U^{*}=1$, to realize an nematic alignment like in Refs. 38, 55, 94, and secondly, asymmetric rods, with $U^{*}=3$, to mimic the swim-off effect observed for colliding bacteria. In the following, we refer to these two situations as models with weak or strong alignment. The self-propulsion is introduced by an effective self-propulsion force $F_{0}$ which is directed along the main rod axis leading to a constant propulsion velocity $v_{0}$ [95]. Hereby, we do not resolve any details about the actual propulsion mechanism.

Colloidal microswimmers move in the low Reynolds number regime, the corresponding overdamped equations of motion for the positions and orientations are

$$
\begin{aligned}
\mathbf{f}_{\mathcal{T}} \cdot \partial_{t} \mathbf{r}_{\alpha}(t) & =-\nabla_{\mathbf{r}_{\alpha}} U(t)+F_{0} \hat{\mathbf{u}}_{\alpha}(t), \\
\mathbf{f}_{\mathcal{R}} \cdot \partial_{t} \hat{\mathbf{u}}_{\alpha}(t) & =-\nabla_{\hat{\mathbf{u}}_{\alpha}} U(t),
\end{aligned}
$$

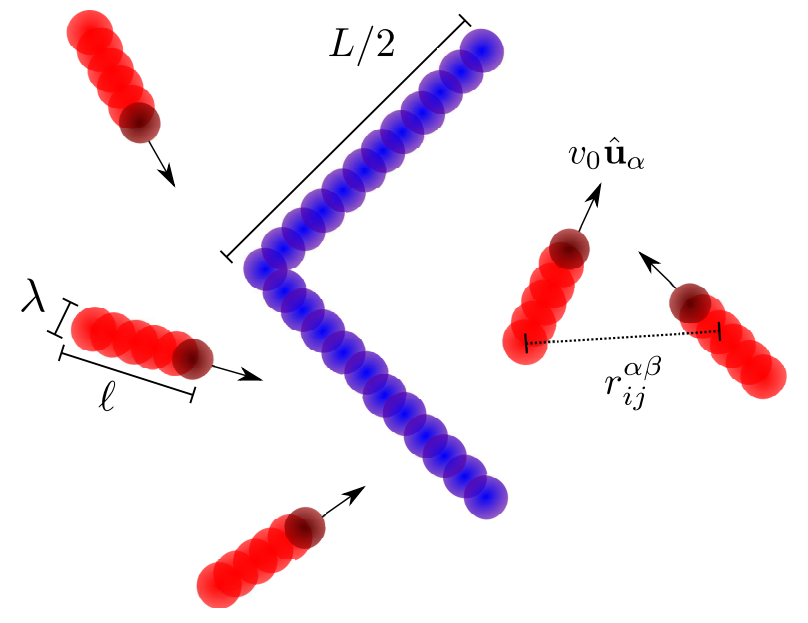

FIG. 1. Sketch of the system of self-propelled rods with aspect ratio $\ell / \lambda$ and an effective self-propulsion velocity $v_{0}$ directed along the main rod axis $\hat{\mathbf{u}}$. The single six Yukawa segments are shown by red circles - a larger interaction prefactor for the first segment of each rod is indicated by darker color. A wedgelike carrier with a short contour length $L$ is shown by blue circles.

in terms of the total potential energy $U=$ $(1 / 2) \sum_{\alpha, \beta(\alpha \neq \beta)} U_{\alpha \beta}+\sum_{\alpha} U_{\alpha<}$ with $U_{\alpha<}$ the potential energy of $\operatorname{rod} \alpha$ with the carrier (the susbcript $<$ is associated with the carrier). The one-body translational and rotational friction tensors for the rods $\mathbf{f}_{\mathcal{T}}$ and $\mathbf{f}_{\mathcal{R}}$ can be decomposed into parallel $f_{\|}$, perpendicular $f_{\perp}$ and rotational $f_{\mathcal{R}}$ contributions which depend solely on the aspect ratio $a=\ell / \lambda$ [96]

$$
\begin{aligned}
\frac{2 \pi}{f_{\|}} & =\ln a-0.207+0.980 a^{-1}-0.133 a^{-2}, \\
\frac{4 \pi}{f_{\perp}} & =\ln a+0.839+0.185 a^{-1}+0.233 a^{-2}, \\
\frac{\pi a^{2}}{3 f_{\mathcal{R}}} & =\ln a-0.662+0.917 a^{-1}-0.050 a^{-2} .
\end{aligned}
$$

The resulting self-propulsion speed $v_{0}=F_{0} / f_{||}$sets to characteristic time unit $\tau=\ell / v_{0}$. We ignore any thermal fluctuations.

According to previous experiments [90] the motion of the submersed carrier will be restricted to translation by using an external magnetic field which keeps the orientation of the carrier fixed. The carrier-swimmer interaction is implemented analogously to the swimmers by tiling the contour length $L$ into Yukawa segments. Most of our data are obtained for $L=26 \ell$ but we do also vary the contour length $L$.

The resulting equation of motion for the carrier is

$$
\mathbf{f}_{<} \cdot \partial_{t} \mathbf{r}_{<}(t)=-\nabla_{\mathbf{r}_{<}} U_{<\alpha}(t),
$$

where $\mathbf{f}_{<}$corresponds to the hydrodynamic friction tensor of the wedgelike carrier, calculated for the spe- 
cific geometry of the carrier using the software package HYDRO++ 97, 98.

We use a square simulation box with area $A=$ $(3 L / \sqrt{2})^{2}$ and periodic boundary conditions in both directions. The total number of swimmers is determined by $N=A \phi / \lambda \ell$, where $\phi$ is a dimensionless packing fraction.

\section{BULK BEHAVIOUR}

Let us start with the characterization of the emergent dynamical states for both considered situations in absence of the carrier. As suitable order parameters we use the averaged swimming speed $\left\langle v_{\alpha}\right\rangle / v_{0}$, measured via the mean swimmer displacement during a time step $\Delta t=$ $10^{-3} \tau$, and the enstrophy $\Omega=\frac{1}{2}\left\langle\left|[\nabla \times \mathbf{V}(\mathbf{r}, t)] \cdot \hat{\mathbf{e}}_{z}\right|^{2}\right\rangle$ for a velocity field $\mathbf{V}(\mathbf{r}, t)$ coarse-grained in space over three swimmer lengths. The results are shown in Figs. 2(a),(b) and show qualitatively the same behavior for both situations, though the achieved values for $\left\langle v_{\alpha}\right\rangle / v_{0}$ and $\Omega$ are slightly higher for swimmers with less alignment. In agreement with experiments [42, 99], we can distinguish between three dynamical states as a function of increasing swimmer density. For low swimmer packing fractions, $\phi \lesssim 0.25$, the average swimmer velocity is $\left\langle v_{\alpha}\right\rangle \gtrsim 0.6 v_{0}$ due to small amount of collisions, leading to a dilute state. For larger densities $0.25 \lesssim \phi \lesssim 0.75$ the velocity is almost constant and the system reveals a large enstrophy $\Omega$ for both systems. Since the enstrophy is a convenient indicator for bacterial turbulence [42, 49], we will refer to this state as turbulent. For high densities $0.75 \lesssim \phi$ the system becomes dynamically jammed, $\left\langle v_{\alpha}\right\rangle \lesssim 0.5 v_{0}$. Using the equal-time spatial velocity autocorrelation function, we can determine the typical swirl radius $R$ for various swimmer concentrations by its first minimum [49]. Hereby, in case of the asymmetric particle model, the typical swirls size is larger, see Fig. 2(c).

To quantify the influence of the body shape asymmetry on the (collective) motion of the swimmers, we study the parallelism of the coarse-grained velocity field and the similarly coarse-grained orientation field $\mathbf{U}(\mathbf{r}, t)$ using the coefficient $C$

$$
C=\frac{\langle\cos \theta\rangle-2 / \pi}{1-2 / \pi}
$$

where $\theta$ is the angle between both fields, see Fig. $2(d)$. Perfectly parallel fields lead to $C=1$, while random directions reveal $C=0$, hence $\langle\cos \theta\rangle=2 / \pi$ for $\theta \in$ $\{-\pi / 2, \pi / 2\}$. In case of the symmetric rods, the mutual collisions in the dilute regime already form dense aligned clusters, leading to high parallelism of the two fields. With increasing swimmer densities and the emergence of large scaled swirls the coefficient $C$ decreases. As is intuitively expected, the stronger the interaction alignment the larger the parallelism between the two fields.
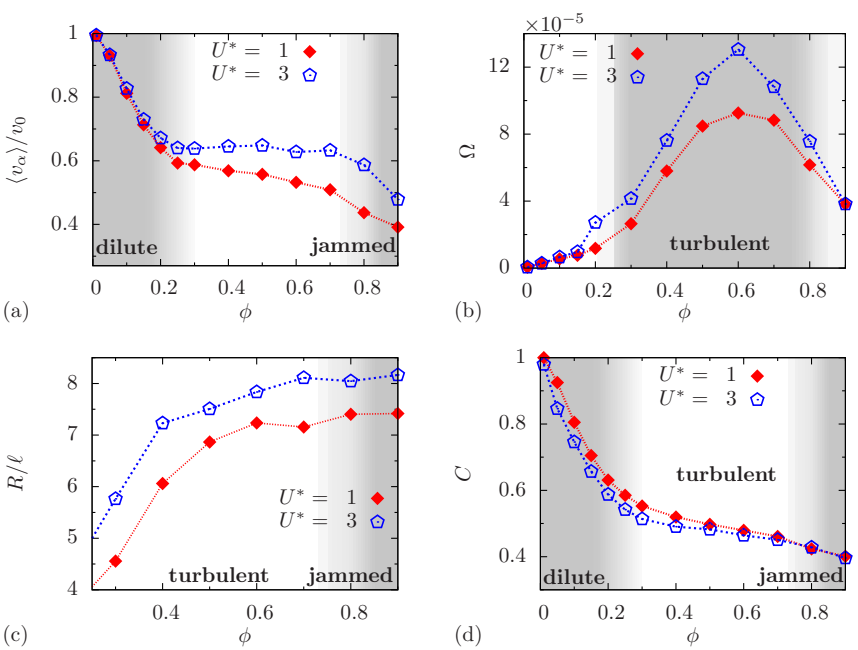

FIG. 2. Comparison of different bulk quantities for system with weak (open symbols) and strong (filled symbols) alignment. (a) Averaged reduced swimmer velocity $\left\langle v_{\alpha}\right\rangle / v_{0}$, (b) mean vorticity $\Omega$, (c) typical reduced swirl size $R / \ell$, and (d) alignment coefficient $C$ as a function of swimmer packing fraction. Shaded areas indicate the emergent dynamical states.

\section{TRANSPORT OF A WEDGELIKE CARRIER}

\section{A. Transport efficiency}

Now, we study the transport efficiency $v / v_{0}$ of a wedgelike carrier for both alignment situations. Due to the symmetry of the wedge any averaged directed motion perpendicular to the apex will vanish. In the apex direction there is no such symmetry and due to rectification the carrier will propagate along this direction. The resulting transport efficiency $v / v_{0}$ is shown in Fig. 3 for two selected contour lengths of the carrier.

While the maximal transport efficiency in around 0.25 for the asymmetric particles, as confirmed by experiment [90], the efficiency for symmetric swimmer is larger $v / v_{0} \approx 0.35$. Moreover, the characteristic density for which the transport is optimal is vastly different: it occurs at low densities in the dilute regime for strong alignment but is significantly shifted towards the turbulent regime for weak alignment. However, both aliment conditions reveal an almost constant efficiency in the turbulent regime. Finally, if the active fluids jam, the carrier velocity clearly decreases as well.

\section{B. Optimization of wedge shape}

We now study the carrier geometry leading to maximal transport efficiency. First of all, we choose for both situations the density which showed the highest transport and vary the apex angle for a fixed contour length $L=26 \ell$, see Fig. 4(a). Clearly, there has to be an optimal angle 


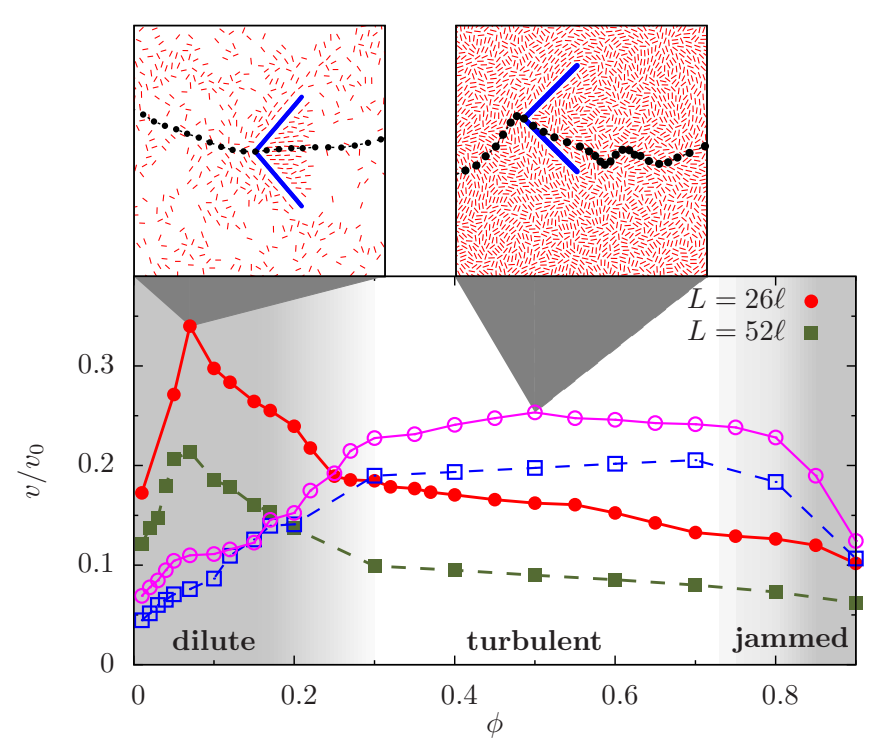

FIG. 3. Transport efficiency for various swimmer densities and two contour lengths of the carrier, $L=26 \ell$ (circles) and $L=52 \ell$ (squares). Filled symbols correspond to weak and open ones to strong alignment. The dynamical states are indicated by shaded areas. The insets show the temporal progress of the carrier position indicated by the dotted line.
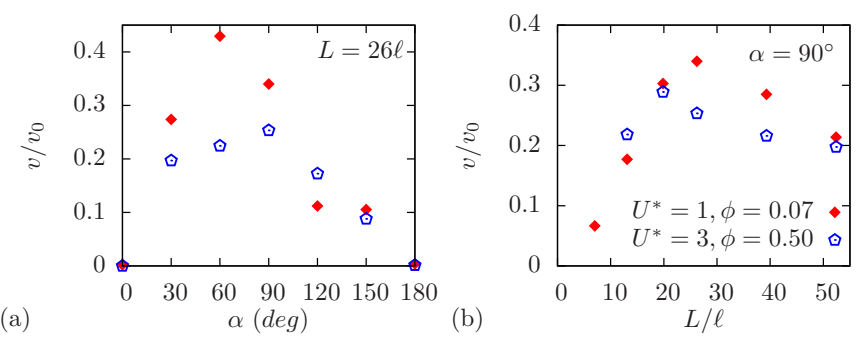

FIG. 4. Transport speed for (a) varied apex angle and fixed contour length $L=26 \ell$ and (b) varied length and fixed apex angle $\alpha=90^{\circ}$ for both situations at a fixed given swimmer packing fraction $\phi$.

between zero and 180 degrees as these two extreme cases do not lead to any transport at all. As a result, the optimal wedge for weak alignment has an apex angle $\alpha=90^{\circ}$ while the optimal angle in the strong alignment model is around $\alpha=60^{\circ}$.

Complementarily, we then have modified the length for a given apex angle $\alpha=90^{\circ}$, see Fig. 4(b). We find an optimal length of $L \approx 20 \ell$ in the weak alignment model and a larger length of $L \approx 26 \ell$ in the strong alignment model.

\section{Transport mechanism}

First of all, we recapitulate the transport mechanisms for the weak alignment model which has been recently explained by swirl shielding inside the carrier [90]. When turbulence sets in, there is a shielding of turbulent fluctu-

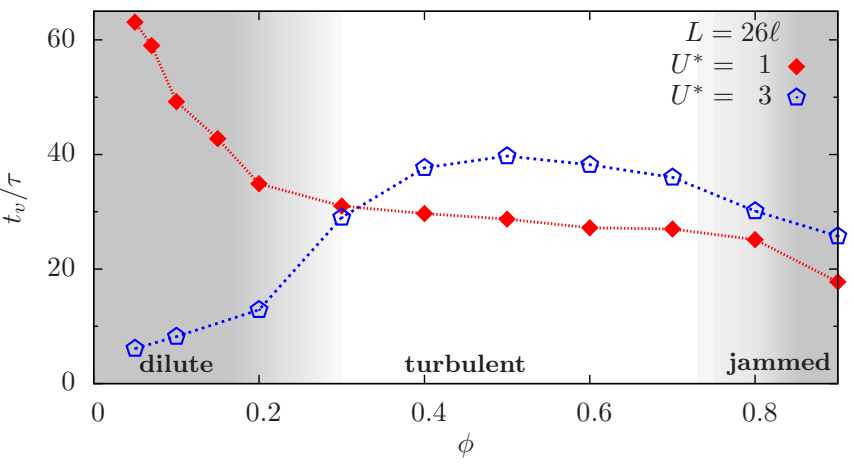

FIG. 5. Velocity correlation time $t_{v} / \tau$ for both particle models, a carrier with contour length $L=26 \ell$ and varied swimmer packing fraction.

ation near the walls of the carrier. Due to the wedgelike geometry this shielding is more pronounced inside the carrier than outside and thus a shielded area near the cusp emerges. Swimmers are trapped inside this area for a long time and are able to push and thereby transport the wedge since they are rectified by the carrier. This process is limited by the flipping processes of the pushing microswimmers inside the wedge. The latter gives rise to fluctuations in the carrier velocity which are characterized by a typical correlation time scale $t_{v}$. This time $t_{v}$ is set by the decay of the normalized and shifted carrier velocity autocorrelation function defined as

$$
C_{v}(t)=\frac{\left\langle v\left(t_{0}\right) v\left(t_{0}+t\right)\right\rangle-\langle v\rangle^{2}}{\left\langle v^{2}\right\rangle-\langle v\rangle^{2}} .
$$

A numerical fit reveals that this quantity decays as $\exp \left(-t / t_{v}\right)$ from which the typical correlation time $t_{v}$ can be extracted. The results are plotted in Fig. 5 for the two alignment situations considered in this work. There is no such swirl shielded area outside the wedge as the swirls can sweep all swimmers away. This imbalance after all pushes the carrier forward. Clearly, this mechanism is valid for both alignment situations in the turbulent state. However, the transport efficiency achieved (see again Fig. 3) is larger for weak alignment since the typical swirl size is larger than in the strong alignment case (see again Fig. 2) and therefore the corresponding swirlshielded area is larger leaving more space for pushing microswimmers. As a consequence of the swirl shielding concept, the optimal transport is achieved when the apex width of the carrier is comparable to the typical swirl size, $L=2 \sqrt{2} R \approx 21 \ell$, see again Fig. 4 .

For strong alignment interactions, the swirl-shielding concept is overwhelmed by another mechanism which occurs already in the dilute regime. Figure 6 shows the local density and the swimmer orientation (by plotting the average $\langle\cos \varphi\rangle$ around the carrier). The density exhibits a "hot spot" near the cusp and a depleted zone in the wake of the carrier. Due to the directed transport the carrier acts like a bulldozer and accumulates swimmers in its front. The intensity plot for the swimmer orienta- 


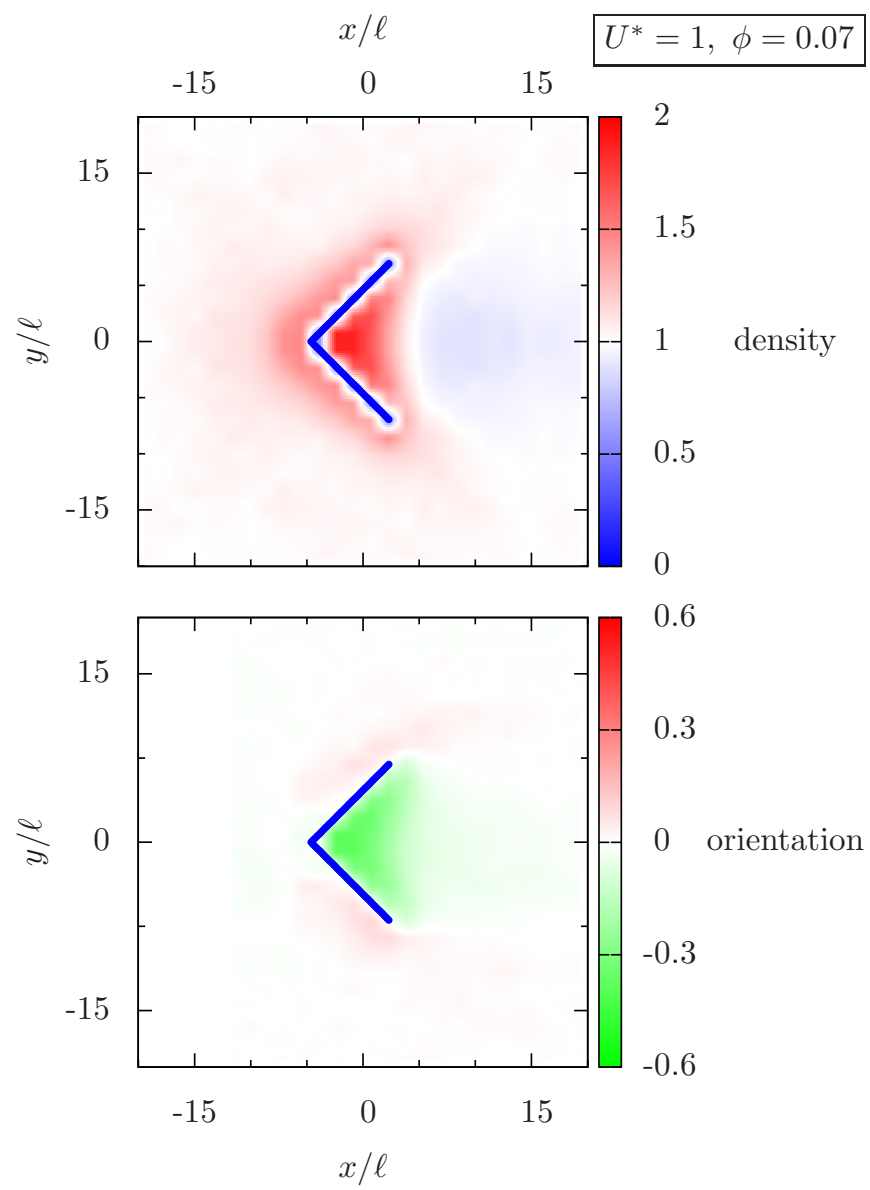

FIG. 6. Intensity plots for (top) the local swimmer density around the carrier and (bottom) the averaged swimmer orientations, using $\langle\cos \varphi\rangle$ for the situation of strong alignment and a packing fraction $\phi=0.07$.

tion shows a clear rectification of swimmers within the wedge even in the wake, where a huge smectic cluster is formed, see left inset of Fig. 3. This cluster is very stable as indicated by a large correlation time $t_{v}$ in the carrier velocity, see again Fig. 5, leading to persistent straight motion of the carrier.

To supplement this picture, we finally present data for the local average density in front of the carrier $\left(\phi_{f}\right)$ and in its wake $\left(\phi_{w}\right)$ in Fig. 7. The emergent smectic cluster in the wake of the carrier for the strong alignment model leads to $\phi_{f} / \phi_{w} \rightarrow 0$ for small swimmer densities, see Fig. 7(a) which supports the strong pushing efficiency. When collective motion starts in the bulk, the ratio $\phi_{f} / \phi_{w}$ gets larger than unity implying that the transport efficiency is decreased. For the weak alignment model this ratio is always larger than unity, see Fig. [7(b). This can be confirmed by experiments on Bacillus subtilis and a microwedge with a contour length $L=52 \ell$ [90], see Fig. 7(c). We can predict this ratio by a simple scaling argument in the dilute regime. The carrier velocity is $v \sim v_{0} \phi$ and the achieved directed motion leads to a density gradient $\left[v \sim D \nabla \phi\right.$, with $\left.D \sim v_{0} \ell / \phi_{w}\right]$. According
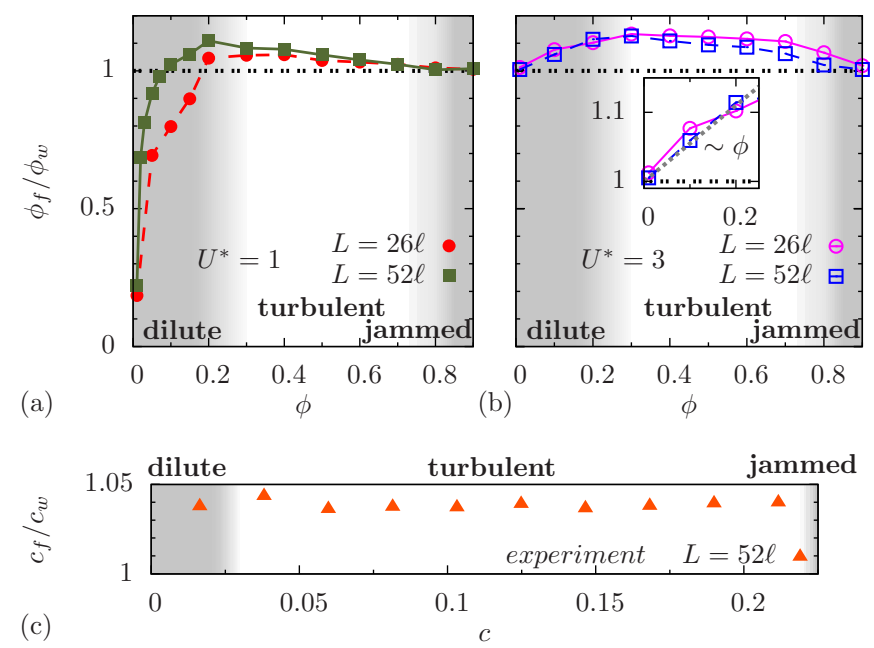

FIG. 7. Comparison of the density around the carrier using the ratio $\phi_{f} / \phi_{w}$, i.e. the ratio of the two densities in the front and in the wake for both interaction models, the two selected contour length as before and varied swimmer packing fractions $\phi$. The inset in (b) shows a close up for the dilute state and a linear dependence of the ratio on the packing fraction $\phi$. (c) Experimentally obtained concentration ratio $c_{f} / c_{w}$, with $c$ the three-dimensional volume fraction.

to this, we can approximate the resulting density ratio for a moving carrier by $\phi_{f} / \phi_{w}-1 \sim \phi$, which is shown in the inset of Fig. 7(b).

To summarize, there are two different mechanisms at work for optimal carrier transport, namely swirl shielding for weak aligning interactions and a large polar pushing cloud of swimmer for strong alignment interactions. The first occurs in the turbulent regime while the latter is in the dilute regime.

\section{CONCLUSION}

In conclusion, we have demonstrated that the actual transport speed of a passive microwedge ("carrier") immersed into an active bath depends on the aligning properties of the individual microswimmers. For strong aligning interactions, as realized for artificial rod-like microswimmers, a polar oriented cloud in the wake of the carrier pushes the carrier forward. Conversely, for interaction without strong alignment, as realized for bacterial swimmers, the most efficient transport occurs in the turbulent state of the active bath and is caused by swirl depletion. Our results were obtained by computer simulations and can be verified in experiments. In particular, experiment with artificial microswimmers exhibiting a strong aligning interaction are highly desirably to test the predicted picture of a polar-ordered wake.

Future work should focus on different shapes of carriers like $L$-particles or $C$-particles which have been considered theoretically [80, 93] but should be realized in experiments. Finally, the carrier transport can be possi- 
bly used as building block to fabricate more complicated micro- and nanomachines steered by an active bath.

\section{ACKNOWLEDGMENTS}

We thank Borge ten Hagen for helpful discussions. A.K. was supported by the ERC Advanced Grant IN-
TERCOCOS (Grant No. 267499) and H.L. by the SPP 1726 of the DFG. Work by A.S. and I.S.A. was supported by the U.S. Department of Energy (DOE), Office of Science, Basic Energy Sciences (BES), Materials Science and Engineering Devision.
[1] P. Romanczuk, M. Bär, W. Ebeling, B. Linder, and L. Schimansky-Geier, Eur. Phys. J. Spec. Top. 202, 1 (2012).

[2] M. E. Cates, Rep. Prog. Phys. 75, 042601 (2012).

[3] M. C. Marchetti, J. F. Joanny, S. Ramaswamy, T. B. Liverpool, J. Prost, M. Rao, and R. A. Simha, Rev. Mod. Phys. 85, 1143 (2013).

[4] I. S. Aranson, Physics-Uspekhi 56, 79 (2013).

[5] A. Sokolov, I. S. Aranson, J. O. Kessler, and R. E. Goldstein, Phys. Rev. Lett. 98, 158102 (2007).

[6] V. B. Shenoy, D. T. Tambe, A. Prasad, and J. A. Theriot, Proc. Natl. Acad. Sci. U.S.A. 104, 8229 (2007)

[7] S. Schmidt, J. van der Gucht, P. M. Biesheuvel, R. Weinkamer, E. Helfer, and A. Frey, Europ. Biophys. J. 37, 1361 (2008).

[8] J. Schwarz-Linek, C. Valeriani, A. Cacciuto, M. E. Cates, D. Marenduzzo, A. N. Morozov, and W. C. K. Poon, Proc. Natl. Acad. Sci. U.S.A. 109, 4052 (2012)

[9] M. Polin, I. Tuval, K. Drescher, J. P. Gollub, and R. E. Goldstein, Science 325, 487 (2009).

[10] J. S. Guasto, K. A. Johnson, and J. P. Gollub, Phys. Rev. Lett. 105, 168102 (2010).

[11] R. Ma, G. S. Klindt, I. H. Riedel-Kruse, F. Jülicher, and B. M. Friedrich, Phys. Rev. Lett. 113, 048101 (2014).

[12] I. H. Riedel, K. Kruse, and J. Howard, Science 309, 300 (2005)

[13] B. M. Friedrich and F. Jülicher, New J. Phys. 10, 123035 (2008).

[14] D. M. Woolley, Reproduction 216, 259 (2003).

[15] T. Vicsek and A. Zafeiris, Phys. Rep. 517, 71 (2012).

[16] M. Ballerini, N. Cabibbo, R. Candelier, A. Cavagna, E. Cisbani, I. Giardina, V. Lecomte, A. Orlandi, G. Parisi, A. Procaccini, M. Viale, and V. Zdravkovic, Proc. Natl. Acad. Sci. U.S.A. 105, 1232 (2008)

[17] Y. Katz, K. Tunstrøm, C. C. Ioannou, C. Huepe, and I. D. Couzin, Proc. Natl. Acad. Sci. U.S.A. 108, 18720 (2011)

[18] J. Buhl, D. J. T. Sumpter, I. D. Couzin, J. J. Hale, E. Despland, E. R. Miller, and S. J. Simpson, Science 312, 1402 (2006)

[19] A. John, A. Schadschneider, D. Chowdhury, and K. Nishinari, Phys. Rev. Lett. 102, 108001 (2009).

[20] J. Zhang, W. Klingsch, A. Schadschneider, and A. Seyfried, J. Stat. Mech. 2012, P02002 (2012)

[21] D. Helbing, I. Farkas, and T. Vicsek, Nature 407, 487 (2000)

[22] J. L. Silverberg, M. Bierbaum, J. P. Sethna, and I. Cohen, Phys. Rev. Lett. 110, 228701 (2013).

[23] A. Ghosh and P. Fischer, Nano Lett. 9, 2243 (2009).

[24] R. Dreyfus, J. Baudry, M. L. Roper, M. Fermigier, H. A. Stone, and J. Bibette, Nature 437, 862 (2005)
[25] P. Tierno, R. Golestanian, I. Pagonabarraga, and F. F. Sagues, J. Phys. Chem. B 112, 16525 (2008).

[26] I. S. M. Khalil, H. C. Dijkslag, L. Abelmann, and S. Misra, Appl. Phys. Lett. 104, 223701 (2014)

[27] W. F. Paxton, K. C. Kistler, C. C. Olmeda, A. Sen, S. K. St. Angelo, Y. Cao, T. E. Mallouk, P. E. Lammert, and V. H. Crespi, J. Am. Chem. Soc. 126, 13424 (2004)

[28] S. Fournier-Bidoz, A. C. Arsenault, I. Manners, and G. A. Ozin, Chem. Commun. , 441 (2005)

[29] J. R. Howse, R. A. L. Jones, A. J. Ryan, T. Gough, R. Vafabakhsh, and R. Golestanian, Phys. Rev. Lett. 99, 048102 (2007).

[30] J. Palacci, C. Cottin-Bizonne, C. Ybert, and L. Bocquet, Phys. Rev. Lett. 105, 088304 (2010)

[31] R. Kapral, J. Chem. Phys. 138, 020901 (2013)

[32] S. Sanchez, A. A. Solovev, S. M. Harazim, and O. G. Schmidt, J. Am. Chem. Soc. 133, 701 (2011).

[33] H.-R. Jiang, N. Yoshinaga, and M. Sano, Phys. Rev. Lett. 105, 268302 (2010)

[34] G. Volpe, I. Buttinoni, D. Vogt, H.-J. Kümmerer, and C. Bechinger, Soft Matter 7, 8810 (2011).

[35] F. Kümmel, B. ten Hagen, R. Wittkowski, I. Buttinoni, R. Eichhorn, G. Volpe, H. Löwen, and C. Bechinger, Phys. Rev. Lett. 110, 198302 (2013)

[36] S. Thutupalli, R. Seemann, and S. Herminghaus, New J. Phys. 13, 073021 (2011).

[37] Y. Yang, V. Marceau, and G. Gompper, Phys. Rev. E 82, 031904 (2010).

[38] F. Ginelli, F. Peruani, M. Bär, and H. Chaté, Phys. Rev. Lett. 104, 184502 (2010)

[39] X. Chen, X. Dong, A. Be'er, H. L. Swinney, and H. P. Zhang, Phys. Rev. Lett. 108, 148101 (2012).

[40] A. Sokolov and I. S. Aranson, Phys. Rev. Lett. 109, 248109 (2012)

[41] D. Saintillan and M. J. Shelley, Phys. Fluids 20, 123304 (2008)

[42] H. H. Wensink, J. Dunkel, S. Heidenreich, K. Drescher, R. E. Goldstein, H. Löwen, and J. M. Yeomans, Proc. Natl. Acad. Sci. U.S.A. 109, 14308 (2012)

[43] K.-A. Liu and L. I, Phys. Rev. E 88, 033004 (2013)

[44] I. Aranson, Physics 6, 61 (2013).

[45] M. Abkenar, K. Marx, T. Auth, and G. Gompper, Phys. Rev. E 88, 062314 (2013).

[46] A. Rabani, G. Ariel, and A. Be'er, PLoS ONE 8, e83760 (2013).

[47] S. Zhou, A. Sokolov, O. D. Lavrentovich, and I. S. Aranson, Proc. Natl. Acad. Sci. U.S.A. 111, 1265 (2014).

[48] E. Lushi, H. Wioland, and R. E. Goldstein, Proc. Natl. Acad. Sci. U.S.A. 111, 9733 (2014)

[49] H. H. Wensink and H. Löwen, J. Phys. Condens. Matter 24, 464130 (2012). 
[50] A. Bricard, J.-B. Caussin, N. Desreumaux, O. Dauchot, and D. Bartolo, Nature 503, 95 (2013)

[51] E. Ferrante, A. E. Turgut, M. Dorigo, and C. Huepe, Phys. Rev. Lett. 111, 268302 (2013)

[52] T. Vicsek, A. Czirók, E. Ben-Jacob, I. Cohen, and O. Shochet, Phys. Rev. Lett. 75, 1226 (1995)

[53] A. Kudrolli, G. Lumay, D. Volfson, and L. S. Tsimring, Phys. Rev. Lett. 100, 058001 (2008)

[54] V. Narayan, S. Ramaswamy, and N. Menon, Science 317, 5834 (2007)

[55] W. Wang, W. Duan, A. Sen, and T. E. Mallouk, Proc. Natl. Acad. Sci. U.S.A. 110, 17744 (2013).

[56] I. S. Aranson, A. Sokolov, J. O. Kessler, and R. E. Goldstein, Phys. Rev. E 75, 040901 (2007).

[57] K. Drescher, J. Dunkel, L. H. Cisneros, S. Ganguly, and R. E. Goldstein, Proc. Natl. Acad. Sci. U.S.A. 108, 10940 (2011).

[58] P. Denissenko, V. Kantsler, D. J. Smith, and J. KirkmanBrown, Proc. Natl. Acad. Sci. U.S.A. 109, 8007 (2012).

[59] A. Guidobaldi, Y. Jeyaram, I. Berdakin, V. V. Moshchalkov, C. A. Condat, V. I. Marconi, L. Giojalas, and A. V. Silhanek, Phys. Rev. E 89, 032720 (2014)

[60] H. H. Wensink and H. Löwen, Phys. Rev. E 78, 031409 (2008)

[61] A. M. Menzel, J. Phys. Condens. Matter 25, 505103 (2013)

[62] C. F. Lee, New J. Phys. 15, 055007 (2013).

[63] J. Elgeti and G. Gompper, Europhys. Lett. 101, 48003 (2013).

[64] Y. Fily, A. Baskaran, and M. F. Hagan, arXiv:1402.5583 (2014).

[65] M. B. Wan, C. J. Olson Reichhardt, Z. Nussinov, and C. Reichhardt, Phys. Rev. Lett. 101, 018102 (2008).

[66] J. Tailleur and M. E. Cates, Europhys. Lett. 86, 60002 (2009).

[67] A. Pototsky, A. M. Hahn, and H. Stark, Phys. Rev. E 87, 042124 (2013).

[68] F. Q. Potiguar, G. A. Farias, and W. P. Ferreira, Phys. Rev. E 90, 012307 (2014)

[69] P. Galajda, J. Keymer, P. Chaikin, and R. Austin, J. Bacteriol. 189, 8704 (2007).

[70] S. E. Hulme, W. R. DiLuzio, S. S. Shevkoplyas, L. Turner, M. Mayer, H. C. Berg, and G. M. Whitesides, Lab Chip 8, 1888 (2008).

[71] J. A. Drocco, C. J. Olson Reichhardt, and C. Reichhardt, Phys. Rev. E 85, 056102 (2012).

[72] I. Berdakin, Y. Jeyaram, V. V. Moshchalkov, L. Venken, S. Dierckx, S. J. Vanderleyden, A. V. Silhanek, C. A. Condat, and V. I. Marconi, Phys. Rev. E 87, 052702 (2013).

[73] A. Costanzo, J. Elgeti, T. Auth, G. Gompper, and M. Ripoll, Europhys. Lett. 107, 36003 (2014).

[74] A. Kaiser, H. H. Wensink, and H. Löwen, Phys. Rev. Lett. 108, 268307 (2012).

[75] L. Restrepo-Pérez, L. Soler, C. S. Martínez-Cisneros, S. Sanchez, and O. G. Schmidt, Lab Chip 14, 1515 (2014).
[76] A. Vagias, R. Raccis, K. Koynov, U. Jonas, H.-J. Butt, G. Fytas, P. Košovan, O. Lenz, and C. Holm, Phys. Rev. Lett. 111, 088301 (2013)

[77] K. C. Leptos, J. S. Guasto, J. P. Gollub, A. I. Pesci, and R. E. Goldstein, Phys. Rev. Lett. 103, 198103 (2009).

[78] G. Miño, J. Dunstan, A. Rousselet, E. Clement, and R. Soto, J. Fluid Mech. 729, 423 (2013)

[79] X.-L. $\mathrm{Wu}$ and A. Libchaber, Phys. Rev. Lett. 84, 3017 (2000)

[80] S. A. Mallory, C. Valeriani, and A. Cacciuto, arXiv:1407.3418 (2014).

[81] A. Kaiser and H. Löwen, J. Chem. Phys. 141, 044903 (2014)

[82] B. ten Hagen, S. van Teeffelen, and H. Löwen, J. Phys. Condens. Matter 23, 194119 (2011).

[83] X. Zheng, B. ten Hagen, A. Kaiser, M. Wu, H. Cui, Z. Silber-Li, and H. Löwen, Phys. Rev. E 88, 032304 (2013).

[84] H. Hess, Annu. Rev. Biomed. Eng. 13, 429 (2011)

[85] L. Angelani, R. DiLeonardo, and G. Ruocco, Phys. Rev. Lett. 102, 048104 (2009).

[86] A. Sokolov, M. M. Apodaca, B. A. Grzyboski, and I. S. Aranson, Proc. Natl. Acad. Sci. U.S.A. 107, 969 (2010).

[87] R. DiLeonardo, L. Angelani, D. DellArciprete, G. Ruocco, V. Iebba, S. Schippa, M. P. Conte, F. Mecarini, F. D. Angelis, and E. D. Fabrizio, Proc. Natl. Acad. Sci. U.S.A. 107, 9541 (2010).

[88] H. Li and H. P. Zhang, Europhys. Lett. 102, 50007 (2013).

[89] L. Angelani and R. D. Leonardo, New J. Phys. 12, 113017 (2010).

[90] A. Kaiser, A. Peshkov, A. Sokolov, B. ten Hagen, H. Löwen, and I. S. Aranson, Phys. Rev. Lett. 112, 158101 (2014)

[91] J. Gachelin, G. Miño, H. Berthet, A. Lindner, A. Rousselet, and E. Clément, Phys. Rev. Lett. 110, 268103 (2013)

[92] T. Kirchhoff, H. Löwen, and R. Klein, Phys. Rev. E 53, 5011 (1996).

[93] H. H. Wensink, V. Kantsler, R. E. Goldstein, and J. Dunkel, Phys. Rev. E 89, 010302 (2014)

[94] S. Ramaswamy, Annu. Rev. Condens. Matter Phys. 1, 323 (2010)

[95] F. Kümmel, B. ten Hagen, R. Wittkowski, D. Takagi, I. Buttinoni, R. Eichhorn, G. Volpe, H. Löwen, and C. Bechinger, Phys. Rev. Lett. 113, 029802 (2014).

[96] M. M. Tirado, C. L. Martinez, and J. G. de la Torre, J. Chem. Phys. 81, 2047 (1984),

[97] J. Garcia de la Torre, S. Navarro, M. C. Lopez Martinez, F. G. Diaz, and J. J. Lopez Cascales, Biophys. J. 67, 530 (1994).

[98] B. Carrasco and J. Garcia de la Torre, J. Chem. Phys. 111, 4817 (1999).

[99] A. Sokolov and I. S. Aranson, Phys. Rev. Lett. 103, 148101 (2009) 